QUARTERLY OF APPLIED MATHEMATICS

VOLUME LXVIII, NUMBER 1

MARCH 2010, PAGE 1

S 0033-569X(09)01170-X

Article electronically published on October 20, 2009

\title{
INTRODUCTION TO THE BROWN UNIVERSITY NONLINEAR WAVE EQUATIONS CONFERENCE PAPERS
}

\author{
BY \\ YAN GUO \\ (Division of Applied Mathematics, Brown University)
}

On May 8-11, 2008, a conference on Nonlinear Wave Equations was held at Brown University, Providence, to honor Walter A. Strauss on the occasion of his 70th birthday. Walter is one of the pioneers in the field of nonlinear wave equations, and this is a collection of papers dedicated to him by his colleagues and friends.

Walter practically invented the mathematical theory of scattering of nonlinear waves. His early work eventually led to the remarkable conclusion, for a large class of nonlinear wave equations, that every finite-energy wave is a scattering state. He also studied the asymptotic decay and the propagation of singularities of linear waves outside a nontrapping obstacle.

Another line of Walter's work dealt with the global existence of solutions of nonlinear wave equations, especially for dissipative equations and conservative equations. He was among the first to construct solitary wave solutions by using constrained variational methods, which initiated a great deal of activity in nonlinear elliptic equations in unbounded space. He pioneered the use of sharp $L^{p}$ estimates for the study of many types of nonlinear wave equations.

Walter initiated a major effort to understand the stability and instability of solitary waves in conservative systems, and his seminal work set up a general framework for such a theory. He is a leader in the recent flowering of mathematical kinetic theory. He has made fundamental contributions in his study of classical solutions to the Vlasov-Maxwell system as well as the stability theory of equilibria in collisionless plasmas.

In recent years, Walter initiated the study of the steady water wave problem in the presence of vorticity, which has opened up many new lines of investigation.

We thank Constantine Dafermos and Walter Freiberger for their strong support for publishing this collection of papers. We are also very grateful to Madeline Brewster for her continual help. We wish many productive years to come for Walter!

Received December 31, 2008.

(C)2009 Brown University Reverts to public domain 28 years from publication 